\title{
Effect of Scutellaria Baicalensis on Scopolamine-Induced Memory and Cognitive Impairment in Rats
}

\author{
Eunby Go, Hyerin Jeong, and Jiwon Choi
}

\begin{abstract}
Many studies have shown that the steamed root of Polygala tenuifolia (PTW) is effective for improving memory. The purpose of this study is to examine whether PTW extracts improved memory defects caused by $\beta$-amyloid (AMY) in the rats. The effects of PTW on the acetylcholinergic system in the hippocampus were also investigated. Male rats were administered daily of PTW. We conducted the Morris water maze (MWM) test as behavioral assessments. Changes in cholinergic system reactivity were also examined by measuring the immunoreactive neurons of acetylcholinesterase (AChE) in the hippocampus. Daily administration of PTW reduced the escape latency for finding the platform in the MWM. The administration of PTW significantly alleviated memory-associated decreases. The results demonstrated that PTW had a significants neuroprotective effect caused by $\beta$-amyloid in rats. These results suggest that PTW may be useful for improving cognitive functioning by stimulating cholinergic enzyme activities and alleviating inflammatory responses.
\end{abstract}

Index Terms- $\beta$-amyloid, memory, Polygala tenuifolia, memory.

\section{INTRODUCTION}

Since senile dementia of the Alzheimer type and multi-infarct dementia accompanying the increase in aging populations has become a social problem, the development of effective drugs for treating these conditions is vital. Among the traditional Korean natural medicines, Polygala root may improve memory, since some Korean prescriptions containing Polygala root have been used to treat amnesia, neurasthenia, palpitation, and insomnia. Recent studies revealed that Polygala tenuifolia roots are effective for memory impairment in rats and mice, and scopolamine-induced impairment of passive avoidance response was improved by oral administration of water extract of Polygala root in mice [1]. Park et al. also reported that ethanol extract from Polygala root (BT-11) was effective against scopolamine-induced cognitive impairments in passive avoidance response and water maze tests. Tenuifoliside B, one of thesaccharides containing acyl groups isolated from P. tenuifolia, had an ameliorative effect on scopolamine-induced impairment in the performance of passive avoidance tasks in rats [2].

The aim of the present study is to evaluate the ability of PTW to improve learning and memory of rats exposed to

Manuscript received February 24, 2014; revised April 25, 2014.

Eunby Go is with the Genetics, Conversation and Evolutionary, Biology Program, National Institute of Standards and Technology, Seoul, Korea (e-mail: eunbygo@naver.com).

Hyerin Jeong and Jiwon Choi are with the HAFS Genetincs, Evolutionary and Biology Republic of Korea, Korea (e-mail: eunbygo96@hanmail.net, jw3259@naver.com). repeated scopolamine-induced memory deficits as measured by performance on the Morris water maze (MWM) test [3]. Moreover, we also examined how these effects were related to the cholinergic system effects to elucidate the neural mechanisms underlying the memory-enhancing activity of PTW.

\section{MAteriALS AND METHODS}

\section{A. Animals}

Adult male Sprague-Dawley (SD) rats weighing 260-280 g were obtained from Samtaco Animal Co. (Seoul, Korea). The rats were housed in a limited-access rodent facility with up to five rats per polycarbonate cage. The room controls were set to maintain the temperature at $22^{\circ} \mathrm{C} \pm 2{ }^{\circ} \mathrm{C}$ and the relative humidity at $55 \% \pm 15 \%$. Cages were lit by artificial light for $12 \mathrm{~h}$ each day. Sterilized drinking water and standard chow diet was supplied ad libitum to each cage during the experiments. All animal experiments began at least 7 days after the animals arrived.

\section{B. Preparation of the Drugs}

$\beta$-amyloid was purchased from Sigma-Aldrich Co. (MO, USA) and dissolved in saline, respectively. Dried roots of Polygala tenuifolia (PTW) were obtained from Kyung-Dong Market (Seoul, Korea). To obtain boiling water extract of PTW, $120 \mathrm{~g}$ of PTW was added to distilled water, heat-extracted at $100^{\circ} \mathrm{C}$, concentrated with a rotary evaporator (Rotavapor R-124, Buchi, Flawil, Switzerland) and lyophilized with a freeze dryer (Eyela, Tokyo, Japan). The powder form of PTW extract (final yield of 14.16\%) was used after diluting it with deionized $\mathrm{H}_{2} \mathrm{O}$ in every experiment.

\section{Experimental Groups}

The control group following repeated injection of $\beta$-amyloid (AMY group, $n=3$ ). Non-treated normal group (NOR group; $n=3$ ). The PTW treated groups (AMY+PTW group, $n=3$ ). The PTW treatments were given 30 min prior to the injection. The memory-like behavioral responses were measured in all groups using the MWM tests.

\section{Morris Water Maze Test}

Morris water maze apparatus: The MWM test was performed using a polypropylene circular pool (painted white internally, $2.0 \mathrm{~m}$ in diameter and $0.35 \mathrm{~m}$ high). The pool contained water maintained at a temperature of $22 \pm 2^{\circ} \mathrm{C}$. The water was made opaque by adding $1 \mathrm{~kg}$ of skim milk powder. During the MWM test, a platform $15 \mathrm{~cm}$ in diameter was located $1.5 \mathrm{~cm}$ below the water in one of four sections of the pool, approximately $50 \mathrm{~cm}$ from the sidewalls. The pool was surrounded by many cues external to the maze. The pool was 
divided into four quadrants of equal area. A digital camera was mounted to the ceiling above the pool and was connected to a computerized recording system equipped with a tracking program (S-MART: PanLab Co., Barcelona, Spain), which permitted on- and off-line automated tracking of the paths taken by the rats.

Hidden platform trial for acquisition test: The MWM test was initiated on the $8^{\text {th }}$ day after the SRG and SCO administration commenced. The animals received three trials per day. The rats were trained to find the hidden platform, which were remained in a fixed location throughout the test. The trials lasted for a maximum of $180 \mathrm{~s}$, and the time it took to find the submerged platform was recorded each time. The animals were tested three trials per day for 6 days, and they received a 60 -s probe trial on the seventh day. Finding the platform was defined as staying on it for at least $4 \mathrm{~s}$ before the acquisition time of $180 \mathrm{~s}$ ended. If the rat failed to find the platform in the allotted time, it was placed onto the platform for $20 \mathrm{~s}$ and assigned a latency of $180 \mathrm{~s}$. Between one trail and the next, water was stirred to erase olfactory traces of previous swim patterns. The entire procedure took seven consecutive days, and each animal had three training trials per day, with a 30- to 40-min inter-trial interval.

Probe trial for retention test: For the probe trial, each rat was placed into the water diagonally from the target quadrant, and for $60 \mathrm{~s}$, it was allowed to search the water from which the platform had been removed. The time ( $\%$ for total time) spent searching for the platform in the former platform quadrant versus the other three quadrants was measured for each rat.

\section{E. Acethylcholinesterase (AchE) Immunohistochemistry}

For immunohistochemical studies, the brains were removed, post-fixed over-night, and cryoprotected with $20 \%$ sucrose in $0.1 \mathrm{M}$ PBS at $4^{\circ} \mathrm{C}$. Coronal sections $30 \mu \mathrm{m}$ thick were cut through the hypothalamus using a cryostat (Leica CM1850; Leica Microsystems Ltd., Nussloch, Germany). The sections were immunostained for AChE expression using the avidin-biotin-peroxidase complex (ABC) method. Briefly, the sections were rinsed three times for $5 \mathrm{~min}$ each in PBS and then incubated with primary goat anti-AChE antibody (1:2000 dilution; Santa Cruz Biotechnology Inc., California, CA, USA) in PBST (PBS plus $0.3 \%$ Triton X-100) for $72 \mathrm{~h}$ at $4^{\circ} \mathrm{C}$. The sections were washed for $5 \mathrm{~min}$ in PBS and then incubated for $120 \mathrm{~min}$ at room temperature with biotinylated rabbit anti-goat IgG antibody serum for anti-AChE antibody. Secondary antibodies were obtained from Vector Laboratories Co. (Burlingame, CA, USA) and diluted 1:200 in PBST containing $2 \%$ normal serum. To visualize immunoreactivity, the sections were incubated for 90 min in $\mathrm{ABC}$ reagent (Vectastain Elite ABC kit; Vector Labs. Co., Burlingame, CA, USA), washed three times for 5 min in PBS, and incubated in a solution containing 3,3'-diaminobenzidine (DAB; Sigma) and $0.01 \% \mathrm{H}_{2} \mathrm{O}_{2}$ for 1 min. Finally, the tissues were washed in PBS, followed by a brief rinse in distilled water, and mounted individually onto slides. Slides were allowed to air dry and were then cover-slipped. Images were captured using the AxioVision 3.0 imaging system (Carl Zeiss, Inc., Oberkochen, Germany) and processed using Adobe Photoshop (Adobe Systems, Inc.,

\section{San Jose, CA, USA).}

\section{F. RNA Isolation and RT-PCR Analyses}

RNA from the brain was isolated from mice in each group. After excision, samples was quickly stored at $-80{ }^{\circ} \mathrm{C}$ until used. Total RNA was isolated using TRIzol $^{\circledR}$ reagent (GIBCO BRL Co., MD, USA). cDNA was synthesized from total RNA with reverse transcriptase (Takara Co., Shiga, Japan). The expression levels of mRNA were determined by RT-PCR analysis. RT-PCR was performed by using a PTC-100 programmable thermal controller (MJ Research Co., MA, USA). The PCR products were separated on $1.5 \%$ agarose gels, stained with ethidium bromide and the density of each band was analyzed by using an image analyzing system (i-MaxTM; Core Bio System Co., Ltd., Seoul, Korea). The expression levels of the cDNA were determined by calculating the relative density ratio of each band.

\section{G. Statistical Analysis}

The data were expressed as the means \pm SEM. The experimental data were analyzed by one-way ANOVA using SPSS program (Version 13.0, Chicago, USA). Statistical differences among groups were further analyzed by using Tukey's post hoc test. $P$ values $<0.05$ were considered statistically significant.

\section{RESULTS}

\section{A. Effect of PTW on the Water Maze Test}

The NOR group rapidly learned the location of the submerged hidden platform and reached it within $20 \mathrm{~s}$ on day 6 of the trials. The PTW-treated rats also showed a reduction in the escape latency through the training period. The AMY group showed a marked retardation in escape latency, probably due to memory deficits resulting from $\beta$-amyloid-induced impairment of learning and memory [4]. The post-hoc test revealed that rats in the AMY+PTW group had significantly reduced swimming latency compared with subjects in the AMY group (Fig. 1). To examine the spatial memory of rats, performance in the probe trial on day $7 \mathrm{w}$ as analyzed by comparing the percentage of time spent swimming to the expected position of platform [5]. The repeated administration of AMY severely impaired spatial performance in the MWM. Rats in the PTW-treated group spent more time around the platform area than did those in the AMY group. PTW treatment significantly attenuated the scopolamine-induced deficit of learning and memory demonstrated in the water maze.[6] This study indicated that the swimming latency in rats receiving PTW administration was higher than those of $\beta$-amyloid-induced deficit rats used as controls.

\section{B. Effect of PTW on the Acetylcholinesterase (AchE) Histochemistry}

AChE-like immunoreactivity was detected primarily in the cell bodies of hypothalamic regions, including the PVN (Fig. 2). In brains of the AMY group, the numbers of AChE immunoreactive fibers in the PVN were increased. The post-doc comparisons revealed that rats treated with immobilization stress alone showed a greater increase in 
AChE expression, as compared to the NOR group. The AChE expression of the AMY+PTW group was lower than that in the AMY group.

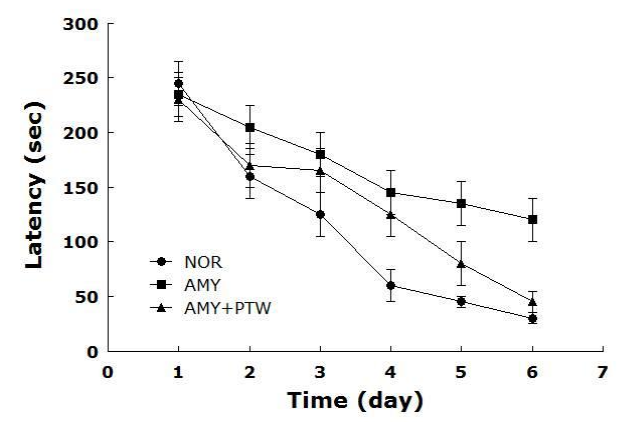

Fig. 1 a). Differences of time between normal group, AMY group and $\mathrm{AMY}+\mathrm{PTW}$ group.

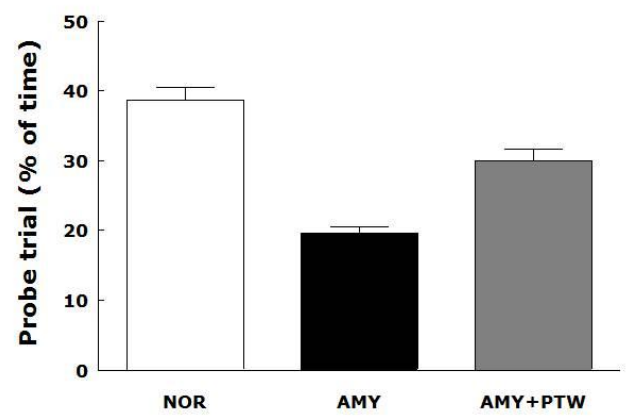

Fig. 1 b). The shorten percentage proportion between 3groups.

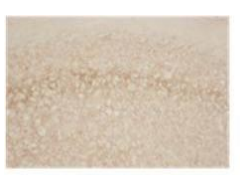

NOR

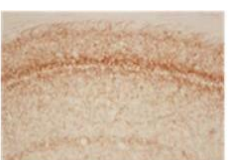

AMY

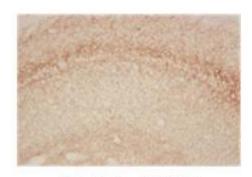

$\mathrm{AMY}+\mathrm{PTW}$
Fig. 2 a). Picture of AChE-like immunoreactivity found in the cell bodies.

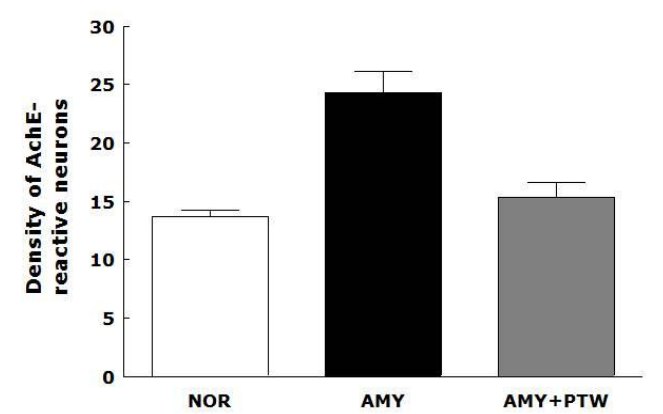

Fig. 2 b). Denstity of AchE-reactive neurons between 3 groups.

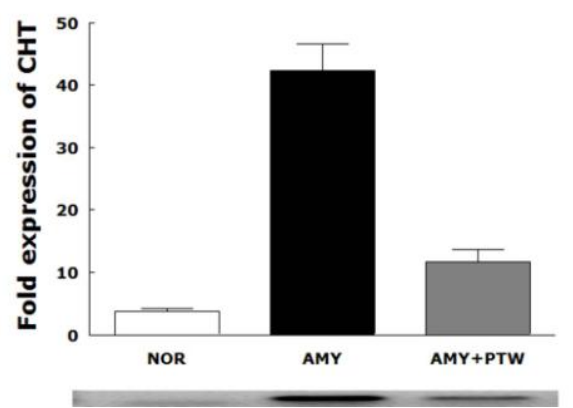

Fig. 3. Levels of CHT.

C. Effect of PTW on CHT mRNA mRNA Expression Levels in the Hippocampus

In order to analyze the cytokine expression, mRNA level in the brain tissue homogenates was determined by using RT-PCR. At the mRNA level CHT was detected, showing in Fig. 3. When the mRNA expression levels in the ear tissue were analyzed, in the NOR group, the mRNA expression levels of CHT showed little change. In the AMY+PTW group, the mRNA expression levels of CHT decreased more than the AMY group.

\section{DISCUSSION}

The present results demonstrated that repeated $\beta$-amyloid-induced dementia produced severe deficits in performances on tests of cognitive functioning as well as corresponding signs of neurodegeneration in the brain, including increased AchE activities in the hippocampus. This result showed that administration of PTW significantly improved learning and memory on the MWM tests and also produced decreased AchE immunoreactivity in the hippocampal areas associated with the $\beta$-amyloid-induced memory impairment of male rats [7].

Acute and chronic administration of PTW in animals has transiently produced several deficits in learning acquisition and short-term memory that are considered to be characteristic of cholinergic deficits in Alzheimer disease [8]. The well-replicated amnesic effect of PTW has been interpreted as a principal consequence of the blockade of post-synaptic muscarinic M1 transmission, leading to disruption in the functioning of the hippocampus in working memory. Thus, the $\beta$-amyloid-induced amnesic model has been widely used to provide a pharmacological model of memory dysfunction for using in screening for agents with potential cognitive-enhancement effects [9].

To confirm the effect of PTW on other types of memory, we performed the MWM test to evaluate spatial learning. When assessing spatial learning and memory by using a rat model, the MWM is considered to be advantageous compared to other types of conventional mazes. Training for spatial memory can be easily achieved by several acquisition trials, and the task does not require strong motivating conditions such as scent, punishment. The MWM is a hippocampus-dependent memory task that is frequently used for examining cognitive deficits and demonstrating permanent spatial learning ability and reference memory in rodents. Animals encode spatial working information during the learning step, which then serves to guide future memory retrieval. During the trial sessions, the PTW group showed significantly reduced escape latency, enhanced cognitive performance, and ameliorated memory deficits associated with AMY.

The expression and activation of $\mathrm{AChE}$ regulated the dynamic concentration of ACh in the cholinergic synapses in the brain [9]. Thus, the hippocampal expression of AChE and its correlation with the $\beta$-amyloid-induced memory impairment rats were examined. PTW also improved cholinergic neurons in the fronto-parietal cortex and CA1 region of the hippocampus and continuously induced increases in ChAT and decreases in AchE activities, which eventually resulted in recovery of the entire cholinergic circulation pathway. It is likely that the observed amelioration in the deficits in spatial learning capability 
demonstrated by the PTW-treated rats was associated with the increasing release of $\mathrm{ACh}$. We thus proposed that the beneficial effects of PTW could be related to increases in central cholinergic functioning.

In this study, we investigate of the cytokine after repeated induction of immobilization stress. For instance, CHT is also playing an important role in depression. CHT over-expression in the brain causes a wide spectrum of pathologies. Therefore, the production of these cytokine was measured. The results showed remarkably and significantly decrease of CHT mRNA expression in the AMY+PTW group compared to the AMY group. The decrease of CHT production was by PTW. Furthermore, these findings suggested that suppression of CHT by PTW produced significant inhibition.

In summary, this result demonstrated that repeated $\beta$-amyloid-induced dementia produced learning and memory in rats, as manifested in their performance on the MWM. However, administration of PTW extracts attenuated these repeated $\beta$-amyloid-induced dementia effects, as indicated by improved cognitive functioning during behavioral tests and increases. Therefore, PTW may possibly be used as an effective agent to prevent cholinergic dysfunction and anti-inflammatory effects such as those observed in Alzheimer disease [10].

\section{CONCLUSION}

The present experiment shows the effect of Polygala Tenuifolia on rats with cognitive impairment, especially the Alzheimer's disease. All age groups are capable of getting the disease, which raises further concerns about the welfare of patients. As high-quality human resource is needed to guarantee their future life, the necessity to study about the cure is rearing its ugly head.

We report here the key route to cure the Alzheimer's disease, which is not yet verified enough to be safe to all conditions. Additionally, the study of PTW's effect on Alzheimer's disease suggests other methods to develop the remedy of the disease. What's more, the study about PTW's effect on the disease can uncover treatment of other cognitive deficiencies.

Yet, there exist some limits whether the effectiveness of PTW will appear equally safe to human beings. Thus, constant and cautious efforts will be needed to make the overall experimenst successful.

\section{REFERENCES}

[1] B. D. Drever, W. G. Anderson, H. Johnson, M. O'Callaghan, S. Seo, D.Y. Choi, G. Riedel, and B. Platt, "Memantine acts as a cholinergic stimulant in the mouse hippocampus," J. Alzheimers. Dis., vol. 12, pp. 319-333, 2007.

[2] D. W. Dickson, S. C. Lee, L. A. Mattiace, S. H. Yen, and C. Brosnan, "Microglia and cytokines in neurological disease, with special reference to AIDS and Alzheimer's disease," Glia, vol. 7, pp. 75-83, 1993.
[3] E. Giacobini, "Long term stabilizing effect of cholinesterase inhibitors in the therapy of Alzheimer's disease," J. Neural. Transm. Suppl., vol. 62, pp. 181-187, 2002.

[4] J. J. Jackson and M. R. Soliman, "Effects of tacrine (THA) on spatial reference memory and cholinergic enzymes in specific rat brain regions," Life Sci., vol. 58, pp. 47-54, 1996.

[5] F. A. Ling, D. Z. Hui, and S. M. Ji, "Protective effect of recombinant human somatotropin on amyloid beta-peptide induced learning and memory deficits in mice," Growth. Horm. IGF. Res., vol. 17, pp. 336-341, 2007.

[6] A. F. Mohamed, K. Matsumoto, K. Tabata, H. Takayama, M. Kitajima, and H. Watanabe, "Effects of Uncaria tomentosa total alkaloid and its components on experimental amnesia in mice: elucidation using the passive avoidance test," J. Pharm. Pharmacol., vol. 52, pp. 1553-1561, 2000.

[7] N. Rothwell, S. Allan, and S. Toulmond, "The role of interleukin 1 in acute neurodegneneration and stroke: pathophysiological and therapeutic implications," J. Clin. Invest., vol. 100, pp. 2648-2652, 1997.

[8] S. Shahidi, A. Komaki, M. Mahmoodi, N. Atrvash, and M. Ghodrati, "Ascorbic acid supplementation could affect passive avoidance learning and memory in rat," Brain Res. Bull., vol. 76, pp. 109-113, 2008.

[9] M. Ohno, T. Yamamoto, and S. Watanabe, "Involvement of cholinergic mechanisms in impairment of working memory in rats following basolateral amygdaloid lesions," Neuropharmacology, vol. 31, 915-922, 1993.

[10] B. D. Pearce and L. T. Potter, "Effects of tetrahydroaminoacridine on M1 and M2 muscarinic receptors," Neurosci. Lett., vol. 88, pp. 281-285, 1988.

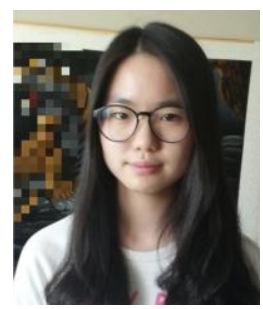

Eunby Go was born in Republic of Korea. She was curious about all the things, especially about the nature. So she attended HAFS and started to write a paper that she studied and researched. But the first time to do anything is always hard to everyone. Although it was hard, she kept working. She studied biology and chemistry. Genetics was her final choice of her major and she tried to discover the secrets of DNA and other genes. In 2012, she continued working on

the project and finished the paper above. She, with 3 fellow workers, is currently working on a paper, concerning $\mathrm{H} 3 \mathrm{~N} 2$ treatment and its sequences.

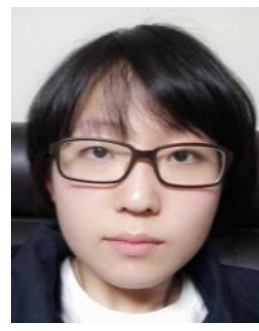

Hyerin Jeong was born in Republic of Korea. In 2002, at high school, she attended several advanced courses, especially chemistry and physics. She later developed especial interest in biology, and is attending advanced biology courses, especially concerning metabolism and genetics. In 2010, Jeong started working on a paper with two more companions, but failed to finish it due to lack of equipment. In 2011, she studied fractals and did a presentation in front of her teachers and colleagues, which received a big applause.

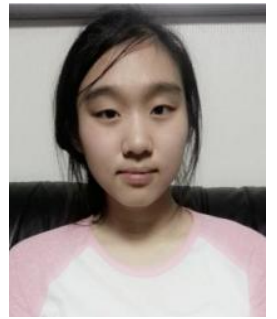

Choi Jiwon was born in Republic of Korea. She had a strong interest and will in studying phenomenon of her surrounding life since she was young. In her early years she joined the SMC program which is a group that studies and experiments wide range of science phenomena from physics, chemistry to biology. After approaching all areas of science, physics, chemistry, geology and biology she learned from the intensive education that she had a keen interest in biology and tried to understand the biology in a deeper way. As having the dream of being a doctor, she was more interested in all kinds of diseases and started to research Alzheimer's disease with her coworkers in Kyung Hee University of Korea. 